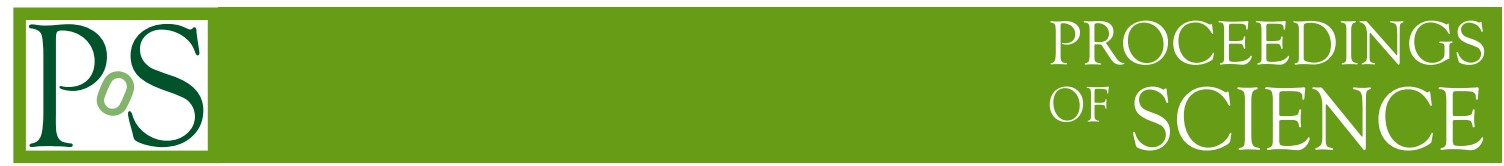

\title{
Higgs to tau tau measurements with CMS
}

\author{
Olena Hlushchenko*i \\ RWTH Aachen University, Physics Institute $3 B$ \\ E-mail: olena.hlushchenko@cern.ch
}

\begin{abstract}
The latest results obtained with CMS data collected at a centre-of-mass energy of $13 \mathrm{TeV}$ targeting Higgs boson decays into tau leptons are shown. The first differential cross section measurement of $H \rightarrow \tau \tau$, as well as the cross-sections measured for individual $\mathrm{ZH}$ and $\mathrm{WH}$ production mechanisms, are presented. The updated limits on anomalous vector boson couplings show no significant deviations from the standard model predictions.
\end{abstract}

European Physical Society Conference on High Energy Physics - EPS-HEP2019 -

10-17 July, 2019

Ghent, Belgium

\footnotetext{
* Speaker.

${ }^{\dagger}$ On behalf of the CMS Collaboration.
} 


\section{Introduction}

In 2012 the discovery of the Higgs boson at a mass of $125 \mathrm{GeV}$ was announced by the ATLAS and CMS collaborations [1, 2, 3]. Following observation of the Higgs boson decay to a pair of tau leptons in 2016 [4] multiple analyses were carried out targeting Higgs boson decays to tau leptons. A brief review of such recent results performed by the CMS Collaboration using LHC data taken at a centre-of-mass energy of $13 \mathrm{TeV}$ during the 2016 and 2017 data taking periods using the CMS detector [5] is presented. These analyses target the main production mechanisms of the Higgs boson via gluon-fusion $(\mathrm{ggH})$, vector boson fusion $(\mathrm{VBF})$ or in association with a vector boson (VH). A closer study of Higgs boson properties constrains anomalous HVV couplings, probing the $C P$ nature of the Higgs boson.

\section{Measurement of Higgs boson production and decay to the $\tau \tau$ final state}

The production cross section for the $H \rightarrow \tau \tau$ process is measured based on the data collected during the 2016 and 2017 data taking periods with a total integrated luminosity of $77.4 \mathrm{fb}^{-1}$ at $\sqrt{s}=13 \mathrm{TeV}$ [6]. Up to $90 \%$ of the expected background contributions were estimated using datadriven methods. The fake-factor [7] method was used to constrain any process where a jet is faking the hadronically decaying tau lepton (W+jets, QCD, $t \bar{t}$, single $t$ and di-boson). Contributions from processes with genuine tau leptons in the final state $(Z \rightarrow \tau \tau, t \bar{t}$ production with two tau leptons in the final state, di-boson, and single $t$ were constrained using $\tau$-embedded techniques described in [8]. The remaining backgrounds arising from events where an electron or muon is misidentified as a hadronic tau lepton decay ( $Z \rightarrow l l, t \bar{t}$, single $t$ and di-boson processes) are estimated using simulation. The analysis categorisation is performed using a neural network (NN) classification algorithm, providing a set of pure categories each targeting one respective signal or background process.

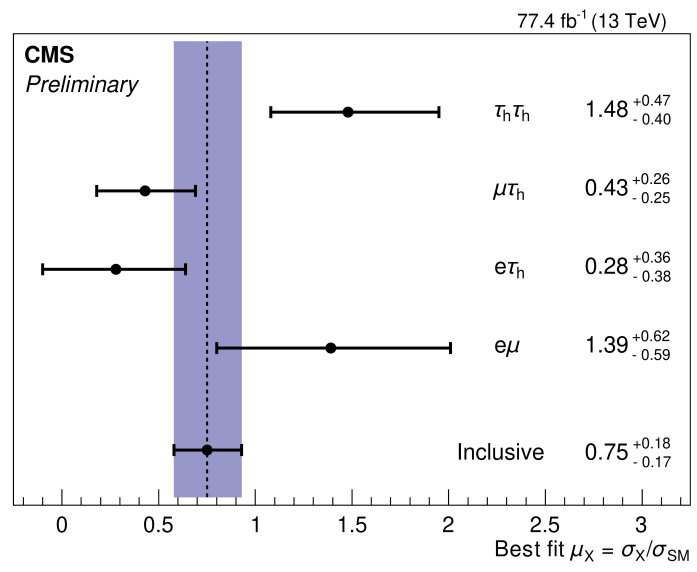

(a)

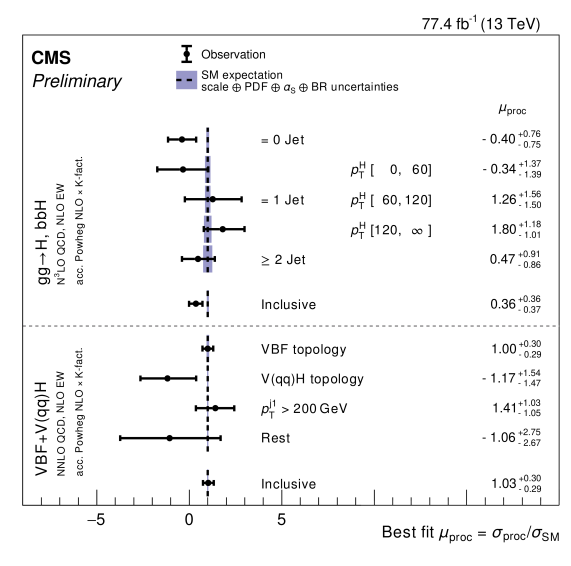

(b)

Figure 1: Constraints on the signal strength parameters obtained from a maximum likelihood fit inclusively and split into individual channels by final state (a), and split by production mechanism and differentially in $p_{T}^{H}$ for the $\mathrm{gg} \rightarrow \mathrm{H}$, bbH and $\mathrm{VBF}+\mathrm{V}$ (qq)H production processes (b) [6]. 
For the inclusive Higgs boson production the product of the production cross-section and branching fraction into tau leptons is measured to be $\sigma^{i n c} B(\mathrm{H} \rightarrow \tau \tau)=2.56 \pm 0.48$ (stat) \pm 0.34 (syst) pb. Following the simplified template cross-section scheme (STXS), as defined by the LHC Higgs Cross Section Working Group [9], when resolved by production modes the products are found to be $\sigma(\mathrm{gg} \rightarrow \mathrm{H}, \mathrm{bbH}) B(\mathrm{H} \rightarrow \tau \tau)=1.11 \pm 0.81$ (stat) \pm 0.78 (syst) pb and $\sigma(\mathrm{VBF}+\mathrm{V}(\mathrm{qq}) \mathrm{H}) B(\mathrm{H} \rightarrow \tau \tau)=0.34 \pm 0.08$ (stat) \pm 0.09 (syst) pb. Here, "gg $\rightarrow \mathrm{H}, \mathrm{bbH}$ " stands for the gluon-fusion and production in association with a $b \bar{b}$ pair, while "VBF+V(qq)H" stands for electroweak qqH production, including both $\mathrm{VBF}$ and $p p \rightarrow V(\rightarrow q \bar{q}) H$ with hadronic $V \rightarrow q \bar{q}$ topologies. The results are presented in terms of constraints on individual signal strength parameters and summarised in Fig.1.

\section{Search for the associated production of the Higgs boson and a vector boson in proton-proton collisions at $\sqrt{s}=13 \mathrm{TeV}$ via Higgs boson decays to tau leptons}

A search for the standard model (SM) Higgs boson produced in association with a W (WH) or a $\mathrm{Z}(\mathrm{ZH})$ boson and decaying into a pair of tau leptons was performed following categorisation targeting respective productions based on three- and four-lepton final states respectively [10]. The used data sample corresponds to an integrated luminosity of $35.9 \mathrm{fb}^{-1}$ and was collected in 2016 at a center-of-mass energy of $13 \mathrm{TeV}$.

The measured signal strength was found to be $\mu=3.39_{-1.54}^{+1.68}$ for $\mathrm{WH}, \mu=1.23_{-1.35}^{+1.62}$ for $\mathrm{ZH}$, and $\mu=2.5_{-1.3}^{+1.4}$ when added (VH).

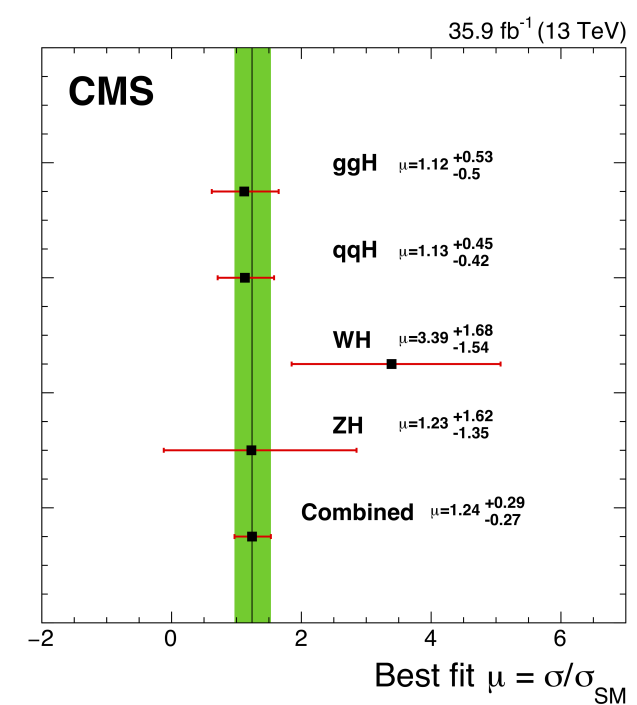

(a)

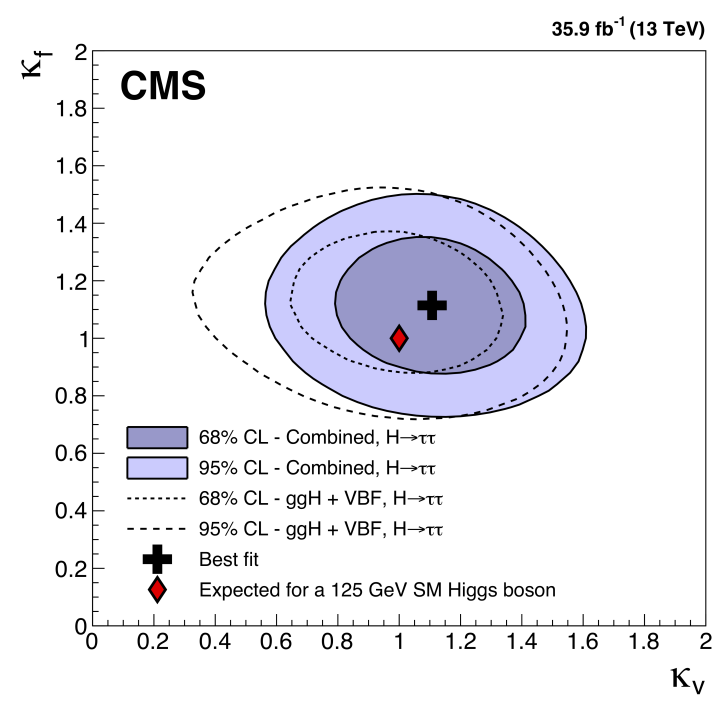

(b)

Figure 2: The observed best-fit signal strengths per Higgs boson production process and their combination as described in [10] (a) and scans in $\kappa_{V}$ and $\kappa_{f}$ space of the negative log-likelihood difference, for $m_{H}=125 \mathrm{GeV}$ (b). The label "Combined" in (b) stands for a combination of the $\mathrm{WH}$ and $\mathrm{ZH}$ targeted analysis while results for the gluon-fusion and vector-boson-fusion analyses are labelled as "ggH + VBF". 
A combination with earlier CMS measurements targeting ggH and VBF production modes for a Higgs boson with a mass of $125 \mathrm{GeV}$ was performed. The best observed (expected) fit signal strength is $\mu=1.24_{-0.27}^{+0.29}\left(1.00_{-0.23}^{+0.24}\right)$, and the observed (expected) significance is 5.5 (4.8) standard deviations as shown in Fig. 2a.

The combination results in additional constraints on the vector boson couplings of the Higgs boson. Measured couplings are consistent with the SM predictions within one standard deviation as seen in Fig. $2 \mathrm{~b}$ providing increased confidence that the Higgs boson couples to tau leptons through a Yukawa coupling as predicted in the SM. The combination allows for the extraction of the signal strengths for the four leading Higgs boson production processes using exclusively $H \rightarrow \tau \tau$ targeted final states, the results of which are largely consistent with the SM. These measurements of the Higgs boson production mechanisms using $H \rightarrow \tau \tau$ decays are the best results to date for the $\mathrm{WH}$ and $\mathrm{ZH}$ associated production mechanisms.

\section{Constraints on anomalous HVV couplings from the production of Higgs bosons decaying to tau lepton pairs}

A study of anomalous HVV couplings was carried out on the data sample collected in the 2016 data taking period and corresponding to an integrated luminosity of $35.9 \mathrm{fb}^{-1}$, using the Higgs boson candidates decaying into pairs of tau leptons and mainly produced in VBF, VH and ggH processes [11].

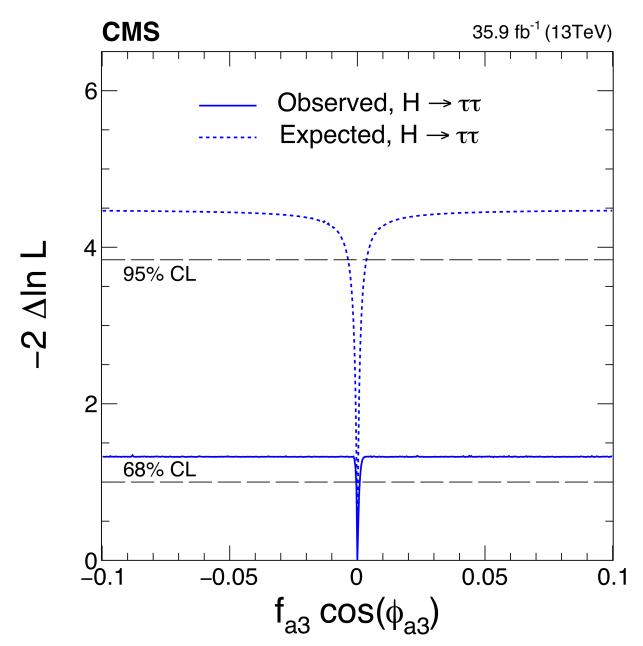

(a)

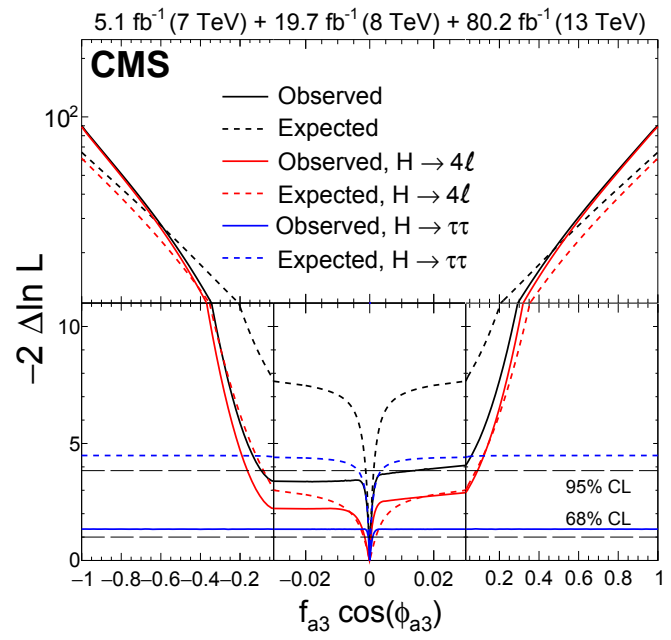

(b)

Figure 3: Observed and expected likelihood scans of $f_{a 3} \cos \left(\phi_{a 3}\right)$ standalone [11] (a) and in combination with $H \rightarrow 4 l$ [16] (b).

Following the same formalism as the one used in the previous publication [12] and theoretical approach described in [13], [14] anomalous interactions of a spin-0 Higgs boson with two spin-1 gauge bosons ( $W W, Z Z, Z \gamma$ and $g g$ ), are parametrized by a scattering amplitude that includes tensor structures allowing to separate scalar( $(0+)$ and pseudoscalar $(0-)$ components. 
The measurement of anomalous interactions relies on the optimal observables formed using the matrix element likelihood approach (MELA) [15]. Constraints derived from the combination of the presented results with those from the $\mathrm{H} \rightarrow 4 l$ [16] decay channel are expressed as effective cross-section fractions and phases for $C P$-violating and $C P$-conserving parameters . The $68 \%$ confidence level constraints are generally tighter than those from previous measurements.

The obtained results are consistent with expectations for the SM Higgs boson as seen in Fig.3.

\section{Conclusion}

The first differential cross-section measurements of $H \rightarrow \tau \tau$ were obtained, using the STXS formalism.

$H \rightarrow \tau \tau$ cross-sections are measured for individual $\mathrm{ZH}$ and $\mathrm{WH}$ production mechanisms as well as in combination with ggH and VBF.

The limits on anomalous couplings were updated. It is found that the analysed dataset does not allow for precise constraints on $C P$ properties.

\section{References}

[1] ATLAS Collaboration, Observation of a New Particle in the Search for the Standard Model Higgs Boson with the ATLAS Detector at the LHC, Phys. Lett. B 716 (2012) 1-29, [doi:10.1016/j.physletb.2012.08.020], [arXiv:1207.7214]

[2] CMS Collaboration, Observation of a new boson at a mass of $125 \mathrm{GeV}$ with the CMS experiment at the LHC, Phys. Lett. B 716 (2012) 30-61, [doi:10.1016/j . phys letb.2012.08.021],, [arXiv:1207.7235]

[3] CMS Collaboration, Observation of a new boson with mass near $125 \mathrm{GeV}$ in pp collisions at $p s=7$ and $8 \mathrm{TeV}, \mathrm{JHEP} 06$ (2013) 081, [doi: 10.1007 / JHEP 06 (2013) 081],, [arXiv:1303.4571]

[4] CMS Collaboration, Observation of the Higgs boson decay to a pair of tau leptons with the CMS detector, CMS-HIG-16-043, [arXiv:1708.00373]

[5] CMS Collaboration, The CMS experiment at the CERN LHC, JINST 3 (2008) S08004, [doi:10.1088/1748-0221/3/08/s08004]

[6] CMS Collaboration, Measurement of Higgs boson production and decay to the $\tau \tau$ final state, CMS-PAS-HIG-18-032

[7] CMS Collaboration, Measurement of the $Z \gamma^{*} \rightarrow \tau \tau$ cross section in pp collisions at $\sqrt{s}=13 \mathrm{TeV}$ and validation of $\tau$ lepton analysis techniques, CMS-HIG-15-007, [arXiv: 1801.03535]

[8] CMS Collaboration, An embedding technique to determine $\tau \tau$ backgrounds in proton-proton collision data, CMS-TAU-18-001, [arXiv:1903.01216]

[9] LHC Higgs Cross Section Working Group Collaboration, Handbook of LHC Higgs cross sections: 4. deciphering the nature of the Higgs sector, [doi:10.23731/CYRM-2017-002], [arXiv:1405.6569]

[10] CMS Collaboration, Search for the associated production of the Higgs boson and a vector boson in proton-proton collisions at $\sqrt{s}=13 \mathrm{TeV}$ via Higgs boson decays to $\tau$ leptons, CMS-HIG-18-007, [arXiv:1809.03590] 
[11] CMS Collaboration, Constraints on anomalous HVV couplings from the production of Higgs bosons decaying to $\tau$ lepton pairs, CMS-HIG-17-034, [arXiv:1903.06973]

[12] CMS Collaboration, Measurements of the Higgs boson width and anomalous HVV couplings from on-shell and off-shell production in the four-lepton final state, [a rXiv:1901.00174]

[13] T. Plehn, D. L. Rainwater, and D. Zeppenfeld, Determining the structure of Higgs couplings at the LHC, Phys. Lett. D 88 (2002) 051801, [doi:10.1103/PhysRevLett.88 . 051801], [arXiv:01053254]

[14] V. Hankele, G. Klamke, D. Zeppenfeld, and T. Figy, Anomalous Higgs boson couplings in vector boson fusion at the CERN LHC, Phys. Rev. D 74 (2006) 095001,

[doi:10.1103/PhysRevD.74.095001], [arXiv:hep-ph/0609075]

[15] Y. Gao et al., Spin determination of single-produced resonances at hadron colliders, Phys. Rev. D 81 (2010) 075022, [doi:10.1103/PhysRevD.81.075022], [arXiv:1001.3396]

[16] CMS Collaboration, Measurements of the Higgs boson width and anomalous HVV couplings from on-shell and off-shell production in the four-lepton final state, [arXiv:1901.00174] 